\section{A Study of Turbulence Simulations Using Finite Difference Lattice Boltzmann Method $^{*}$}

\author{
Daisuke OSHIMA $^{* *}$, Michihisa TSUTAHARA ${ }^{* *}$ and Satoshi MIZUTANI** \\ ${ }^{* *}$ Graduate School of Engineering, Kobe University, \\ Rokkodaimachi, Nada-ku, Kobe-shi, Hyogo, 657-8501 Japan \\ E-mail: tutahara@mech.kobe-u.ac.jp
}

\begin{abstract}
Three-dimensional homogeneous isotropic turbulence is simulated using the finite difference lattice Boltzmann method (FDLBM). First, we analyze the characteristics of numerical viscosity of FDLBM, and present a method in which the numerical viscosity coincides with that of the Navier-Stokes based FDM. Second, we conducted simulations with the Smagorinsky model. In the energy spectrum $\mathrm{E}(\mathrm{k})$ one detects the inertial range $\mathrm{E}(\mathrm{k}) \sim \mathrm{k}^{-5 / 3}$; however, the large $\mathrm{k}$ range presents a persistent energy "tail," which has also been found in direct simulations using conventional lattice Boltzmann scheme. This energy "tail" is is due to generation of non-physical longitudinal noise. However, for high Reynolds number flows this noise is shown to be negligible.
\end{abstract}

Keywords: Computational Fluid Dynamics, Lattice Boltzmann Method, Turbulent Flow, Numerical Viscosity, Incompressible Flow, Large Eddy Simulation

\section{Introduction}

Although the lattice Boltzmann method (LBM) ${ }^{(1)-(7)}$ is different from the methods based on the Navier-Stokes equations, it has attracted attention as a new method of computational fluid dynamics for solving the evolution of the distribution function of virtual particles. The finite difference lattice Boltzmann method (FDLBM) solves the basic equation of the LBM - the discrete BGK equation - by stable finite difference schemes. The FDLBM makes the calculations remarkably stable and can use arbitrary shaped grids independent of the particle motion. Therefore, this method is very effective for practical simulations ${ }^{(8)}$. The authors have shown the effectiveness of this method by calculating the Aeolian tones and sounds emitted from moving bodies ${ }^{(9)}$.

The number of reports of direct turbulence simulation and model simulation using LBM are increasing. However, as far as the authors know, no report of turbulence calculation using FDLBM has been reported. The authors performed a calculation of low Reynolds number isotropic homogeneous turbulence by the FDLBM using an incompressible fluid model, and obtained a good result. However, the result shows a "tail" in which the energy of the large wave number region in the energy spectra is larger than the results from experiments or other numerical simulations. This tail also appears in simulations using conventional $\mathrm{LBM}^{(1)(4)(7)}$. Although this does not raise significant problem for the entire field, the spectra is incorrect. This tail is assumed to be due to the numerical viscosity of higher order as that appearing in the Spectral methods ${ }^{(7)}$.

In this study, we use a turbulence model to show that this tail can be negligible for high Reynolds number turbulence, and investigate the cause of this tail. We also discuss the
${ }^{*}$ Received 19 Sep., 2007 (No. T1-06-0578) Japanese Original : Trans. Jpn. Soc. Mech. Eng., Vol.73, No.727, B (2007), pp.736-742 (Received 25 May, 2006) [DOI: 10.1299/jfst.2.582] 
numerical viscosity that appears in the FDLBM, and compare it with the numerical viscosity in Navier-Stokes equation-based calculations.

\section{The finite difference lattice Boltzmann method}

\subsection{Basic equations}

FDLBM is a computational fluid dynamics tool in which stable finite difference schemes are introduced for particle models used in the LBM. By this method, the grids and the discrete particle velocities can be treated independently. The motions of the particles are described by the distribution function $f_{i}$, which is the number density of the particle having velocity $\mathbf{c}_{i}$.

In this study, we use the following discrete Boltzmann (BGK) equation ${ }^{(10)}$ :

$$
\frac{\partial f_{i}}{\partial t}+c_{i \alpha} \frac{\partial f_{i}}{\partial x_{\alpha}}-a c_{i \alpha} \frac{\partial}{\partial x_{\alpha}} \frac{f_{i}-f_{i}^{(0)}}{\phi}=-\frac{1}{\phi}\left(f_{i}-f_{i}^{(0)}\right)
$$

where subscript $i$ represents the direction of particle translation, $\alpha$ represents the Cartesian co-ordinates, and $f_{i}^{(0)}$ is the local equilibrium distribution function. The term on the right hand side represents the collision of particles, and $\phi$ is the single relaxation time factor. The third term on the left hand side represents the negative viscosity $(a>0)$, which makes the viscosity small for high Reynolds number flows. The value of $a$ can be arbitrary chosen (hereafter $\mathrm{a}=0.5$ ), unless it is too large..

Macroscopic variables are obtained by multiplying the moment of the particle velocity to the equilibrium distribution function, and summing them at each grid point as

$$
\begin{aligned}
& \rho=\sum_{i} f_{i}=\sum_{i} f_{i}^{(0)} \\
& \rho u_{\alpha}=\sum_{i} f_{i} c_{i \alpha}=\sum_{i} f_{i}^{(0)} c_{i \alpha}
\end{aligned}
$$

where $\rho$ is the fluid density and $u_{\alpha}$ is the flow velocity. By defining the density and the momentum by the local distribution function and simultaneously by the equilibrium distribution function, these values are conserved at the collision stage.

In this study, we use a non-thermal incompressible model, and do not consider energy conservation or the internal energy.

\subsection{Three-dimensional fifteen velocity model}

We use a 3-dimensional, 15 velocity (D3Q15) model for incompressible and non -thermal fluids. The local equilibrium distribution function for this model is written as

$$
f_{i}^{(0)}=\rho\left[A_{n}+B_{n} c_{i \alpha} u_{\alpha}+C_{n}\left(c_{i \alpha} u_{\alpha}\right)^{2}+D_{n} u_{\alpha}^{2}\right]
$$

where $n=0,1$, and 2 represent the rest particle, the particle with velocity 2 , and the particle with velocity $\sqrt{3}$, respectively. Therefore the local equilibrium distribution function is expressed by the fluid density and the flow velocity, and in the polynomial form of the flow velocity up to the second order.

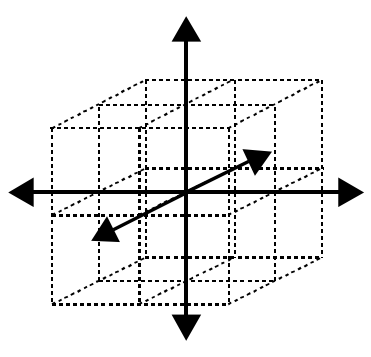

(a) $n=1$

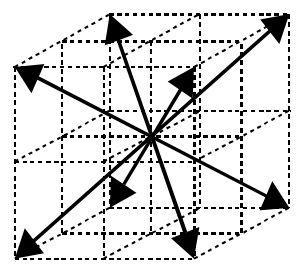

(b) $n=2$

Fig.1 Distribution of particles in the 3D15V model 
The Navier-Stokes equations are recovered by the Chapman-Enskog expansion technique, and the terms in (3) are determined as ${ }^{(4)}$ follows:

$$
\begin{array}{llll}
A_{0}=\frac{1}{23}, & B_{0}=0, & C_{0}=0, & D_{0}=-\frac{7}{24} \\
A_{1}=\frac{1}{23}, & B_{1}=\frac{1}{24}, & C_{1}=\frac{1}{32}, & D_{1}=-\frac{1}{48} \\
A_{2}=\frac{2}{23}, & B_{2}=\frac{1}{12}, & C_{2}=\frac{1}{16}, & D_{2}=-\frac{1}{24} .
\end{array}
$$

The pressure $P$ and the viscosity $\mu$ are given as follows:

$$
\begin{aligned}
& P=\frac{24}{23} \rho, \\
& \mu=\frac{2}{3} \rho(\phi-a) .
\end{aligned}
$$

\section{Large Eddy Simulation}

\subsection{Turbulence model}

We use the Smagorinsky model ("S" model) and the dynamic Smagorinsky model ("DS" model) (11). The S model is a representative, sub-grid scale (SGS) model, and the non-isotropic part of the SGS stress is expressed as

$$
\tau_{\alpha \beta}-\frac{\delta_{\alpha \beta}}{3} \tau_{k k}=-2 v_{t} \bar{S}_{\alpha \beta}
$$

where $\delta_{\alpha \beta}$ is the Kronecker delta and $v_{t}$ is the eddy viscosity. $\bar{S}_{\alpha \beta}$ is the rate of deformation tensor in grid size and is expressed as

$$
\bar{S}_{\alpha \beta}=\frac{1}{2}\left(\frac{\partial \bar{u}_{\alpha}}{\partial x_{\beta}}+\frac{\partial \bar{u}_{\beta}}{\partial x_{\alpha}}\right)
$$

where upper bar represents the variables in grid scale.

In the $\mathrm{S}$ model, the eddy viscosity $v_{t}$ is assumed in the form

$$
\begin{aligned}
& v_{t}=C \Delta^{2}|\bar{S}| \\
& |\bar{S}|=\sqrt{2 \bar{S}_{\alpha \beta} \bar{S}_{\alpha \beta}}
\end{aligned}
$$

where $C$ is the square of the Smagorinsky constant.

In the DS model, $C$ in (8.a) is dynamically determined. Based on the model proposed by Germano et $\mathrm{al}^{(12)}$, and using the calculation procedure by Lilly's method of least squares $^{(13)}, C$ is given by

$$
C=-\frac{\lambda_{m n} M_{m n}}{M_{k l} M_{k l}}
$$

where

$$
\begin{aligned}
& \lambda_{\alpha \beta}=\overline{\bar{u}}_{\alpha} \bar{u}_{\beta}-\tilde{\bar{u}}_{\alpha} \tilde{\bar{u}}_{\beta} \\
& M_{\alpha \beta}=\tilde{\bar{\Delta}}^{2}|\tilde{\bar{S}}| \tilde{\bar{S}}_{\alpha \beta}-\bar{\Delta}^{2}|\bar{S}| \bar{S}_{\alpha \beta} .
\end{aligned}
$$

\subsection{Sub-grid model in FDLBM}

In general, the governing equations are averaged in space. A filter function $G(y)$ is used for averaging, and in the FDLBM the averaging is done using

$$
\bar{f}(x)=\int_{-\infty}^{\infty} G(y) f(x-y) d y .
$$

The filtered discrete BGK equation becomes

$$
\frac{\partial \bar{f}_{i}}{\partial t}+c_{i \alpha} \frac{\partial \bar{f}_{i}}{\partial x_{\alpha}}-a c_{i \alpha} \frac{\partial}{\partial x_{\alpha}} \frac{\overline{f_{i}-f_{i}^{(0)}}}{\phi_{\text {total }}}=-\frac{1}{\phi_{\text {total }}}\left(\overline{f_{i}}-\overline{f_{i}^{(0)}}\right)
$$


where $\phi_{\text {total }}$ is the relaxation time factor. However, this is different from ordinary LBM and a function of time and space. The Navier-Stokes equations are recovered by the Chapman-Enskog procedure, even when the relaxation time factor is a parameter depending on time and space. Therefore, (11) leads to

$$
\begin{aligned}
& \frac{\partial \bar{u}_{i}}{\partial x_{i}}=0 \\
& \frac{\partial \bar{u}_{i}}{\partial t}+\overline{u_{j}} \frac{\partial \bar{u}_{i}}{\partial x_{j}}=-\frac{1}{\rho} \frac{\partial \bar{p}}{\partial x_{i}}+2\left(v+v_{t}\right) \overline{S_{i j}}
\end{aligned}
$$

that are filtered Navier-Stokes equations. For the D3Q15 model, the relaxation time factor $\phi_{\text {total }}$ is given by

$$
\phi_{\text {total }}=\frac{3}{2}\left(v+v_{t}\right)+a
$$

and substituting the eddy viscosity of "S Model" or "DS Model" to $v_{t}$, we obtain $\phi_{\text {total }}$ for each model.

\section{Numerical viscosity of FDLBM}

\subsection{Numerical viscosity term}

It is sometimes effective to use the numerical viscosity for stable calculations. However, the numerical viscosity should be used with care, e.g., if the numerical viscosity is too large, it leads completely different flow for high Reynolds number flows. Here, we consider a scheme in which a weighted numerical viscosity term is added to a fourth-order central difference on a uniform grid. The finite difference formation in the $x$ direction for the distribution function $f_{i}$ is given by

$$
c_{x} \frac{\partial f_{i}}{\partial x}=c_{x} \frac{f_{i-2}-8 f_{i-1}+8_{i+1}-f_{i+2}}{12 \Delta x}+\beta \frac{\left|c_{x}\right|}{12} \frac{f_{i-2}-4 f_{i-1}+6 f_{i}-4 f_{i+1}+f_{i+2}}{\Delta x}
$$

where $\beta=1.0$ corresponds to the third order upwind scheme UTOPIA.

\subsection{Numerical viscosity of discrete BGK equation}

The discrete BGK equation, in which the additional negative viscosity term is eliminated for simplicity, is

$$
\frac{\partial f_{i}}{\partial t}+c_{i \alpha} \frac{\partial f_{i}}{\partial x_{\alpha}}=-\frac{1}{\phi}\left[f_{i}-f_{i}^{(0)}\right]
$$

The convection term of the above equation is discretized by the scheme represented in (14), and the following equation is obtained:

$$
\frac{\partial f_{i}}{\partial t}+c_{i \alpha} \frac{\partial f_{i}}{\partial x_{\alpha}}=-\frac{1}{\phi}\left[f_{i}-f_{i}^{(0)}\right]+\sum_{\alpha} \operatorname{sign}\left(c_{i \alpha}\right) \frac{\beta c_{i \alpha} \Delta x^{3}}{12} \frac{\partial^{4} f_{i}}{\partial x_{\alpha}{ }^{4}}
$$

where the second term on right hand side represents the numerical viscosity or, in this case, the numerical diffusion.

This numerical viscosity for the distribution function $f_{i}$ is proportional to the convection velocity for $f_{i}$ - that is $c_{i \alpha}$. Assuming that $f_{i}$ can be approximated by the local equilibrium distribution function given by (3) and (4), and using the relationship given in $(2 b)$, the numerical viscosity is expressed as

$$
\frac{\partial \mathbf{u}}{\partial t}=\frac{\alpha c \Delta x^{3}}{9} \frac{\partial^{4} \mathbf{u}}{\partial x^{4}}+\frac{\alpha c \Delta y^{3}}{18} \frac{\partial^{4} \mathbf{u}}{\partial y^{4}}+\frac{\alpha c \Delta z^{3}}{18} \frac{\partial^{4} \mathbf{u}}{\partial z^{4}}
$$

where $\mathbf{u}=(u, v, w)$ is the flow velocity vector, and $\Delta x, \Delta y, \Delta z$ are the grid sizes. The variables are non-dimensionalized as follows: 


$$
\begin{aligned}
& \mathbf{u}^{*}=\left(u^{*}, v^{*}, w^{*}\right)=\left(\frac{u}{U_{0}}, \frac{v}{U_{0}}, \frac{w}{U_{0}}\right) \\
& x^{*}=\frac{x}{L}, y^{*}=\frac{y}{L}, z^{*}=\frac{z}{L} \\
& \Delta x^{*}=\frac{\Delta x}{L}, \Delta y^{*}=\frac{\Delta y}{L}, \Delta z^{*}=\frac{\Delta z}{L} \\
& t^{*}=\frac{t U_{0}}{L}
\end{aligned}
$$

where $U_{0}, L$ are the reference velocity and length, respectively.

Equation (17) is non-dimensionalized using (18) and leads to

$$
\frac{\partial \mathbf{u}^{*}}{\partial t^{*}}=\frac{\alpha c \Delta x^{* 3}}{9 U_{0}} \frac{\partial^{4} \mathbf{u}^{*}}{\partial x^{* 4}}+\frac{\alpha c \Delta y^{* 3}}{18 U_{0}} \frac{\partial^{4} \mathbf{u}^{*}}{\partial y^{* 4}}+\frac{\alpha c \Delta z^{* 3}}{18 U_{0}} \frac{\partial^{4} \mathbf{u}^{*}}{\partial z^{* 4}} .
$$

Equation (19) shows that the numerical viscosity is proportional to the ratio of the particle velocity to the flow velocity $c / U_{0}$. The particle velocity is usually considered to be unity, and when the flow velocity is small, the numerical viscosity becomes significant. In LBM, quasi-compressibility appears even for incompressible models, and quasi-sound waves exist (longitudinal waves, but independent of the temperature). The speed of the quasi-sound is $c_{s}=c / \sqrt{3}$, and it is the order of particle velocity. This order estimation will hold for compressible and thermal fluid models. Therefore, the numerical viscosity becomes significant when the flow Mach number is small.

\subsection{Numerical viscosity suitable for FDLBM}

From the above analysis, numerical viscosity becomes relatively large when the flow velocity is small. On the other hand, when the same scheme is applied to the Navier-Stokes equations, the velocity corresponding to the particle velocity, which appears in (16) and (17), is the macroscopic flow velocity $\mathbf{u}=(u, v, w)$. In this case, the numerical viscosity becomes small when the flow velocity is small.

To recover the numerical viscosity for the Navier-Stokes based calculation, the weight of the numerical viscosity $\beta$ should be multiplied by the flow velocity. Equation (17) becomes

$$
\frac{\partial \mathbf{u}}{\partial t}=\frac{\beta u c \Delta x^{3}}{9} \frac{\partial^{4} \mathbf{u}}{\partial x^{4}}+\frac{\beta v c \Delta y^{3}}{18} \frac{\partial^{4} \mathbf{u}}{\partial y^{4}}+\frac{\beta w c \Delta z^{3}}{18} \frac{\partial^{4} \mathbf{u}}{\partial z^{4}} .
$$

This equation is non-dimensionalized by (18), and leads to

$$
\frac{\partial \mathbf{u}^{*}}{\partial t^{*}}=\frac{\alpha u^{*} c \Delta x^{* 3}}{9} \frac{\partial^{4} \mathbf{u}^{*}}{\partial x^{* 4}}+\frac{\alpha v^{*} c \Delta y^{* 3}}{18} \frac{\partial^{4} \mathbf{u}^{*}}{\partial y^{* 4}}+\frac{\alpha w^{*} c \Delta z^{* 3}}{18} \frac{\partial^{4} \mathbf{u}^{*}}{\partial z^{* 4}} \text {. }
$$

Equation (21) shows that the numerical viscosity does not depend on the flow velocity $U_{0}$.

\section{Three-dimensional homogeneous and isotropic turbulence}

\subsection{Calculation parameters}

The calculation domain is a cubic region and a $64 \times 64 \times 64$ uniform grid is used. Disturbance of a sinusoidal form is given as the initial condition, and the Reynolds number, based on the energy of the turbulence and the rate of dissipation, is taken to be 40000 . Time integration is performed by the second order Runge-Kutta method, and the spatial differential is discretized by an ordinary scheme in (14), which refers to Case $1(\beta=0.4)$, and the proposed scheme in (20), which refers to Case 2 . Calculations were performed for $\beta=1.5,2.0$, and 2.5 , and $\beta=2.0$ was selected because this value best fit the results of the Navier-Stokes equations. More precise determination of the value of $\beta$ requires an estimation of the solution by the Navier-Stokes equations, and a detailed discussion will be 
presented in future work. The time increment is $\Delta t=0.018$.

The calculations comparing the finite difference scheme for the Navier-Stokes equation with our results are performed as follows ${ }^{(14)}$. A staggered mesh is employed. For time integration, Adams-Bashforth method is applied to the convection term and Crank-Nicolson method to the viscose term. The spatial differentiation is replaced by the fourth order central finite deference scheme. Other parameters, such as the Reynolds number, are the same as calculated using the FDLBM sub grid model.

The Smagorinsky constant for the $\mathrm{S}$ model is the ordinary value $\mathrm{Cs}=0.173$, and when the eddy viscosity becomes negative, it is chosen as 0 for the DS model.

\subsection{Results}

The energy spectra at time step 10000 are shown in Fig. 2 using the S model, and in Fig. 3 using the DS model, in which the vertical axis represents the energy and the horizontal axis represents the wave number on a logarithmic scale. Figure 2 shows that the energy dissipation is large in the large wave number region because, in Case 1, the numerical viscosity depends on the Mach number. On the other hand, the spectra in Fig. 3 agree well with those from calculations using the Navier-Stokes equations. The energy is proportional to the power of $5 / 3$ of the wave number. However, the tail appears in the FDLBM calculations for the maximum wave number region, even though it is not that significant.

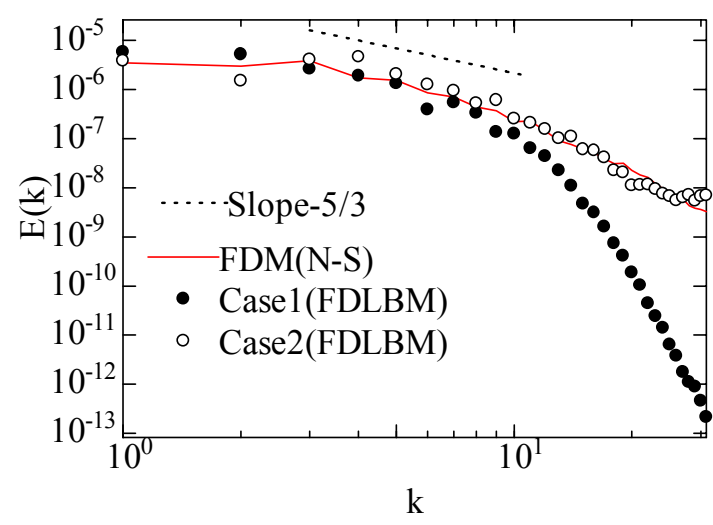

Fig. 2 Energy spectra with the S Model (time step = 10000).

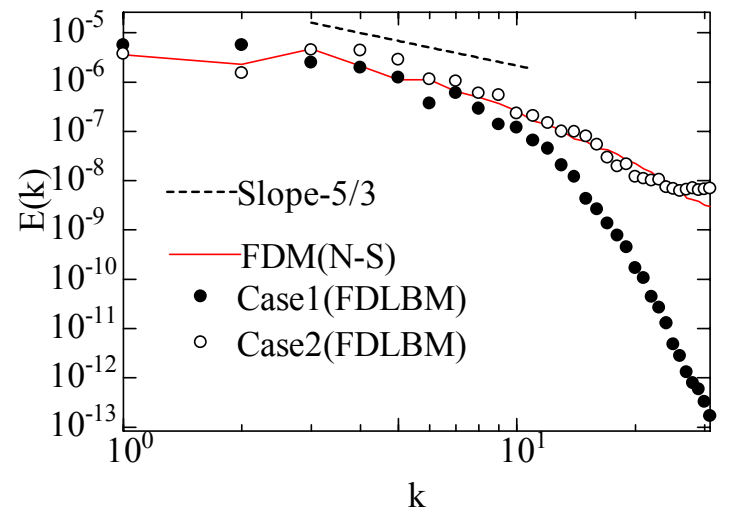

Fig. 3 Energy spectra with the DS Model (time step $=10000)$. 


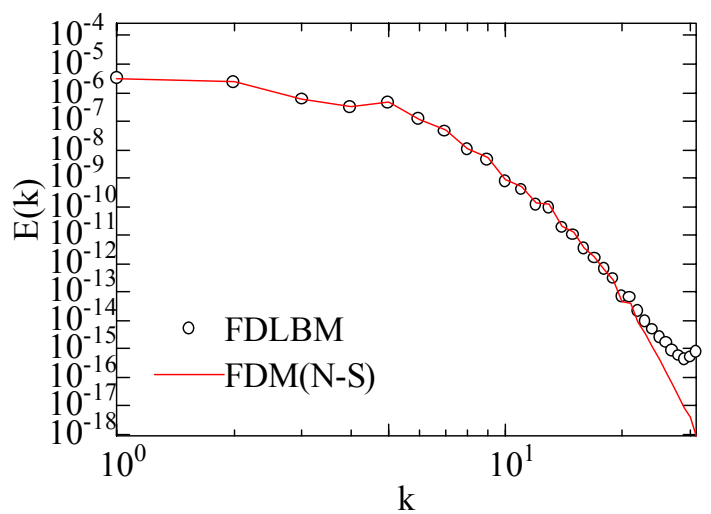

Fig. 4 Energy spectra with the DS Model for Low Reynolds number turbulence (time step = 10000).

\subsection{Reynolds number dependence of the tail}

For better comparison, the results are shown from direct simulation without turbulence, and from the FDLB and the Navier-Stokes equations for low Reynolds number turbulence. The parameters of the calculation are the same as the LES simulation; however, now the Reynolds number is 400. The large tail that appears in FDLBM calculations is different from the high Reynolds number turbulence simulation. This tail does not depend on grid size or time increment. It is independent of the accuracy of calculation, and appears not only for turbulence calculations but also for laminar flow calculation.

The details of the tail are studied by laminar flow to obtain an analytical solution.

\section{Verification of generation of lateral waves by laminar flow calculation}

\subsection{Calculation conditions}

The calculation domain is a cubic region with sides of $2 \pi$, and a $32 \times 32 \times 32$ uniform grid is used. A periodic boundary condition is applied to all three directions, and the initial condition is $(u, v, w)=(0.1 \sin (z), 0,0)$. The Reynolds number, based on the side length and maximum velocity, is 62 . Time integration is done by the second order Runge-Kutta method, and the fourth order central finite difference scheme is employed for space discretization. The time increment is $\Delta t=0.015$, and the reference density is $\rho_{0}=1.0$.

\subsection{Results}

Figures 5, 6, and 7 show the distributions of the density $\rho$, the velocity component in the $y$ direction is $v$, and the velocity component in the $z$ direction is $w$, along $(\pi, \pi, z)$, respectively. Although in Fig. 5 a periodic fluctuation of the density in the $z$ direction is visible, it is very small. In addition, there seems to be periodic fluctuation in $v$, as shown in Fig. 6, but it is extremely small and noisy, and can be considered calculation noise. On the other hand, the velocity component $w$, in the $z$ direction, in which the flow velocity $u$ has a velocity gradient, shows a periodic fluctuation with the same wave number as the density. The wave number of the fluctuation is 2 -twice the initial disturbance $u=0.1 \sin (z)$.

Figure 8 shows the $w$ distributions at time steps 20, 30, and 40, and from this the variation is found to be a stationary wave, with a period of 208 , in time steps. Therefore the period $T$ is given by

$T=\Delta t \times$ timestep $=3.12$,

and the propagation speed of the longitudinal wave is obtained by 


$$
c=\frac{\omega}{k}=\frac{2 \pi}{T k} \approx 1.0 \text {. }
$$

This speed of the wave is the same as the particle velocity in the $z$ direction in this model. This longitudinal wave (density variance) is not a sound wave whose phase velocity is Cs $=$ $\mathrm{c} / \sqrt{3}$, due to the quasi compressibility of the model, but a non-physical disturbance, propagating with the particle velocity $c$.

Figure 9 shows the time histories of $w$ at $(\pi, \pi, 7 / 8 \pi)$ for Reynolds numbers 16 , 31 , and 62 . The variation decreases as the Reynolds number increases.

The tail appearing in the energy spectra can be considered to be generated by the weak longitudinal waves. In general, non-physical longitudinal waves are found to be generated by particle motion in the direction of the flow velocity changes. Turbulent flow fields are constructed by velocities in various directions, and the longitudinal waves propagate complicatedly in various directions, and construct the tail in turbulence calculations of the LBM and FDLBM.

Comparing $u$ and $w$, however, it is found to be $w / u \approx O\left(10^{-5}\right)$, and, although the shape of energy spectra becomes different at a high wave number region, this tail is not significant, even for low Reynolds number turbulence. For high Reynolds number flows, the amplitude of the longitudinal waves becomes much smaller, and the effect of the tail can be negligible in LES calculations for high Reynolds number turbulence.

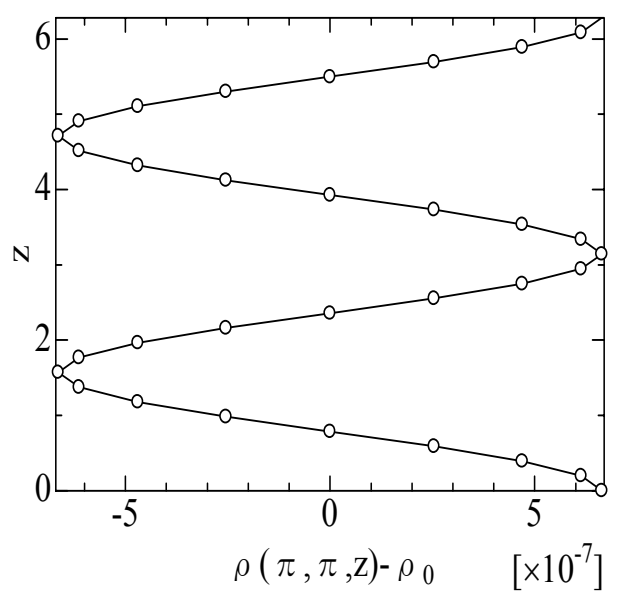

Fig. 5 Distribution of density at $\mathbf{x}=(\pi, \pi, z)$.

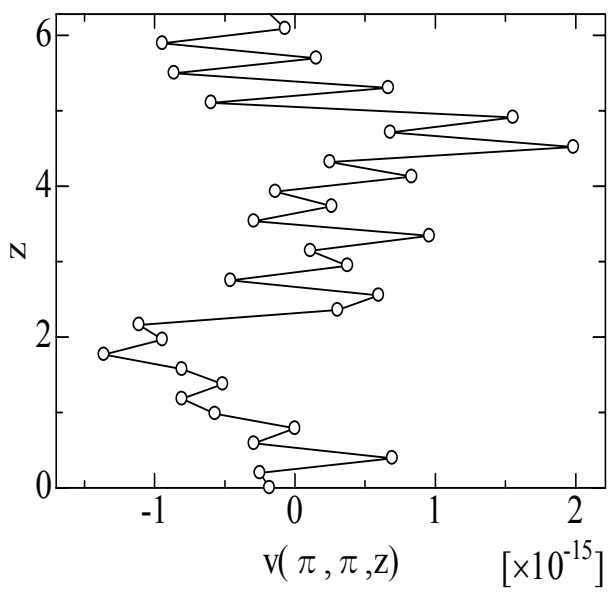

Fig. 6 Distribution of velocity $\mathrm{v}$ at $\mathbf{x}=(\pi, \pi, \mathrm{z})$. 


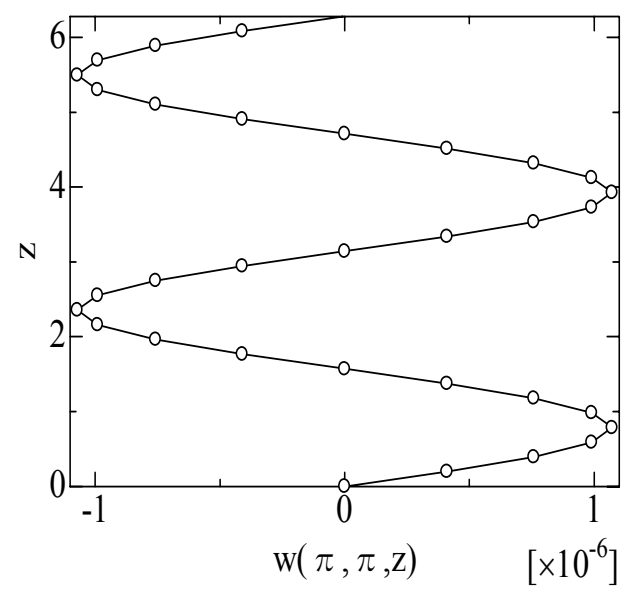

Fig. 7 Distribution of velocity $\mathrm{w}$ at $\mathbf{x}=(\pi, \pi, \mathrm{z})$.

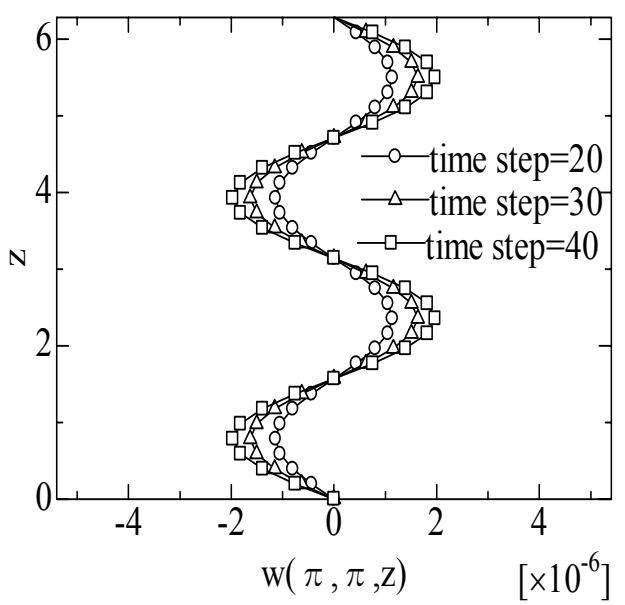

Fig. 8 Distribution of velocity $\mathrm{w}$ at $\mathbf{x}=(\pi, \pi, \mathrm{z})$.

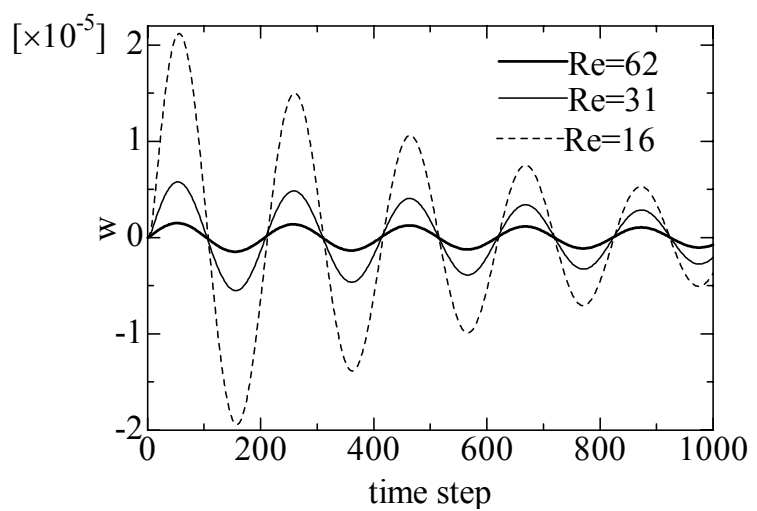

Fig. 9 Time evolution of density at $\mathbf{x}=(\pi, \pi, 7 / 8 \pi)$.

\section{Conclusions}

Three-dimensional isotropic homogeneous turbulence is calculated with the sub-grid model using FDLBM. The numerical viscosity, which strongly depends on the flow velocity is eliminated by introducing a numerical viscosity. With this numerical viscosity, the spectra in which the energy is proportional to the $-5 / 3$ power of the wave number in the inertial sub-range are obtained. By a laminar-flow calculation, non-physical longitudinal waves exist in the FDLBM simulations, and these waves seem to appear as the tail in turbulence 
simulations.

The results of three-dimensional simulation for uniform and isotropic turbulence with sub-grid model in the FDLBM agree with that from the FDM of the Navier-Stokes equation, because the tail is not significant for high Reynolds number flows. The sub-grid FDLBM model can be applied to other turbulent flow simulations of a high Reynolds number.

\section{References}

(1) Qian, Y. H., Succi S. and Orszag, S. A., Recent Advances in Lattice Boltzmann Computing, Ann. Rev. of Comp. Phy. III, D. Stauffer ed. World Scientific, (1995), pp.195-242.

(2) Rothman, D. H. and Zalenski, S., Lattice-Gas Cellular Automata, Cambridge University Press (1997).

(3) Chopard, B. and Droz, M., Cellular Automata Modeling of Physical Systems, Cambridge University Press (1998).

(4) Chen, S. and Doolen G.D., Lattice Boltzmann method for fluid flows, Ann. Rev. Fluid Mech., Ann. Rev. Inc., (1998), pp.329-364.

(5) Tsutahara, M. et al., Lattice Gas and Lattice Boltzmann Methods, Corona Publishing Co. Ltd. (1999) in Japanese.

(6) Wolf-Gladrow, D.A., Lattice-Gas Cellular Automata and Lattice Boltzmann Models, Lecture Notes in Mathematics, Springer (2000).

(7) Succi S., The lattice Boltzmann Equation for Fluid Dynamics and Beyond, Oxford (2001).

(8) Cao, N. S., Chen, S., Jin, S., Martinez, D., Physical symmetry and lattice symmetry in the lattice Boltzmann method, Phys. Rev., E, 1997; 55: R21-R24.

(9) Tsutahara, M., et al., T., Direct Simulation of Acoustic Waves by the Finite Difference Lattice Boltzmann Method, Transactions of the Japan Society of Mechanical Engineers, Series B, Vol.69, No.680 (2003), pp.89-95

(10) Tsutahara, M., et al., A Study of New Finite Difference Lattice Boltzmann Method, Transactions of the Japan Society of Mechanical Engineers, Series B, Vol. 68, No.665 (2002), pp.15-21

(11) Kajishima, T., Numerical Simulation of Turbulent Flows, Yokendo Publishing Co. Ltd. (1999) in Japanese.

(12) Germano, M., Piomelli, U., Moin, P., and Cabot, W. H., A dynamic subgrid-scale eddy viscosity model, Phys. Fluids A, Vol.3 No.7 (1991), pp.1760-1765

(13) Lilly, D.K., A proposed modification of the Germano subgrid-scale closure model, Phys. Fluids A, Vol. 4 No.4 (1992), pp.633-635.

(14) http://www-fluid.mech.eng.osaka-u.ac.jp/ kajisima/ 